\title{
Heterogeneous Researchers in a Two-Sector Representative Consumer Economy*
}

\author{
Orlando Gomes ${ }^{\dagger}$
}

Contents: 1. Introduction; 2. A Multiple Research Projects Two-Sector Model; 3. Dynamics and SteadyState Properties; 4. Growth-Paths: A Numerical Example; 5. Discussion - Heterogeneous Researchers and Endogenous Fluctuations; 6. Conclusions; A. Proof of Propositions; B. Numerical Example Time Trajectories.

Keywords: Heterogeneous agents; Bounded rationality; Optimal control; Research activities; Volatility and chaos.

JEL Code: C61; O32; 033.

Research activities have uncertain outcomes. The question asked in this paper is whether or not this uncertainty can be a central piece on the explanation of long run consumption growth paths. More specifically, we inquire how the existence of different research projects, with different degrees of uncertainty, contributes to unpredictable consumption growth paths. The proposed scenario is a two-sector representative consumer model with researchers that invest in different innovation projects. There is heterogeneity in terms of risk associated to research programs (researchers invest in projects with the same expected outcome but different volatility). This difference in volatility, combined with an adaptive learning - bounded rationality rule, implies an aggregate index of technology and a consumption growth rate that do not present a predictable pattern over time.

As actividades de investigação produzem resultados incertos. A questão colocada neste artigo é se esta incerteza pode ser uma peça central na explicação das trajectórias de crescimento do consumo no longo prazo. Mais especificamente, pergunta-se como a existência de diferentes projectos de investigação, com diferentes graus de incerteza associados, contribui para trajectórias de crescimento do consumo que não são passíveis de previsão. O cenário

\footnotetext{
*Financial support from the Fundação Ciência e Tecnologia, Lisbon, is grateful acknowledged, under the contract No POCTI/ECO/48628/2002, partially funded by the European Regional Development Fund (ERDF). I also acknowledge the important comments made by an anonymous referee and the helpful assistance of the journal's editors. The usual disclaimer applies.

†Escola Superior de Comunicação Social [Campus de Benfica do Instituto Politécnico de Lisboa; 1549-014 Lisboa, Portugal] and Unidade de Investigação em Desenvolvimento Empresarial [UNIDE/ISCTE]. ogomes@escs.ipl.pt
} 
proposto é um modelo de consumidor representativo de dois sectores com investigadores que investem em diferentes projectos de inovação. Existe heterogeneidade ao nível do risco associado aos programas de investigação (os investigadores investem em projectos com o mesmo resultado esperado mas diferente volatilidade). A diferença na volatilidade, combinada com uma regra de aprendizagem adaptativa - racionalidade limitada, implica um índice agregado de tecnologia e uma taxa de crescimento do consumo que não apresentam um padrão previsível ao longo do tempo.

\section{INTRODUCTION}

Heterogeneity regarding economic agents beliefs and behavior is an important field in today's economic research. The most influential work at this level respects to the explanation of asset prices fluctuations. Starting with the work of Brock and Hommes (1998), several authors have tried to explain how the co-existence of fundamentalist traders and technical analysts contributes to a random and hardly predictable time series for asset prices. In this kind of asset pricing models, heterogeneity combined with an adaptive belief system allows to find time paths for asset prices that are erratic, that is, where periods of low volatility and high volatility alternate, where volatility clustering is evidenced and where some important empirical features about financial markets can be mimetized. Some important work concerning asset pricing heterogeneous agents was developed in Brock et al. (2001), Hommes et al. (2002), Gaunersdorfer et al. (2003), Azariadis and Kaas (2002), Chiarella and He (2002), Kurz and Schneider (1996), Kurz (1997a), Kurz and Beltratti (1997) and Kurz and Motolese (2001).

Heterogeneity and adaptive beliefs are also an influential line of thought of contemporary macroeconomics, mainly in what concerns expectations and learning mechanisms. The most important references at this level are, on one hand, the bounded rationality approach of Sargent (1993) and the discussion of learning mechanisms by Evans and Honkapohja (2001). Other authors, like Barucci (1999), Nourry and Venditti (2001), Tuinstra and Wagener (2003) and Negroni (2003) study stability conditions of macroeconomic models with heterogeneous agents.

Heterogeneity analysis is today extended to a large number of economic issues. Besides asset pricing and macroeconomic stability, different individual behavior or expectations serves as a means to explain exchange rate fluctuations [De Grauwe and Grimaldi (2002)], economic growth [Maliar and Maliar (2001), Becker and Tsyganov (2002)] or monetary policy [Kurz et al. (2003)].

The model to develop in this paper combines, as the previous references, a mechanism of bounded rationality and learning with the notion of agent heterogeneity. This model is an endogenous growth two sector model where a representative consumer maximizes utility. The source of heterogeneity is in technology generation [as in Kurz et al. (2003)] and not in consumer preferences as it became usual in this kind of model [it is the case of Becker and Tsyganov (2002)] - the representative consumer structure continuous to hold. Under such a scenario we observe that different risk in R\&D activities can explain long run consumption growth rates that are erratic and impossible to predict.

Research activities are risky by nature; nevertheless, some are riskier than others. Individuals or firms engaged in research activities choose their research projects between a set of possibilities with equal expected returns but different volatilities. Sometimes, the ones that bet in higher risk activities are the most successful ones; in other occasions, the ones that play safe attain the best result. This constant switching in terms of the best performance strategy is the key ingredient for the non predictable long run time paths to encounter. Because technology producers cannot change from one research activity to another instantly (we consider a learning bounded rationality mechanism) there will always be a certain number of agents choosing some research investment strategy; this share changes according 
to accumulated past results concerning the innovation activity, such that in certain periods of time it increases and in others it declines.

The fundamental result is that heterogeneity in one economic sector is a source of randomness and unpredictability for the whole economic system. The production of final goods may not be associated to unpredictable outcomes, at least not in the same extent as the generation of knowledge, but final goods time trajectories become erratic trajectories in the moment that we consider a technological level that is determined by the dynamics of a heterogeneous agents - bounded rationality research sector.

The remainder of the paper has the following contents. Section 2 characterizes the main features of the model. A two-sector model is constructed, where the first sector generates a homogeneous final good that can be indistinctly consumed or used in subsequent periods as capital, and the second sector is an R\&D sector. Section 3 assumes a steady state scenario with no volatility. In this case the properties of the model are the ones common to the Romer-Jones endogenous growth model. In section 4 the dynamic analysis of the model is pursued through a numerical example. We understand with this example that a same set of parameters and initial values implies time paths of the most important economic aggregates that change each time the example is run. Section 5 discusses why is the present analysis relevant; in particular, it addresses the macroeconomic business cycles / endogenous fluctuations literature to motivate both theoretically and empirically the undertaken analysis. We argue that our model is a special case of a real business cycle framework, where technology shocks are replaced by a systematic effort of generating technical knowledge (an effort that comes from a large set of independent, autonomous and heterogeneous researchers). Finally, section 6 makes a few final comments. Two appendixes are also included: appendix A concerns to the proof of the propositions presented in section 3 , while appendix $B$ is destined to the presentation of the most important time paths of the numerical example in section 4 .

\section{A MULTIPLE RESEARCH PROJECTS TWO-SECTOR MODEL}

We begin by assuming a discrete time infinite horizon utility maximization problem for a given representative consumer. In this problem, variable $c_{t}$ denotes the level of real consumption in each time moment, $\rho>0$ is a constant discount factor and $U\left(c_{t}\right)$ will represent the utility function. The utility function respects the following assumptions,

i) $\mathrm{U}$ is continuous, concave and smooth (infinitely many times continuously differentiable);

ii) $\theta>1$ is a concavity parameter of the utility function that obeys the condition $U^{\prime}=c_{t}^{-\theta}$.

The optimal control problem consists on the maximization of the flow of utility functions in expression (1),

$$
\sum_{t=0}^{\infty} U\left(c_{t}\right) \cdot \frac{1}{(1+\rho)^{t}}
$$

The maximization problem is constrained by the economy's production possibilities. Following the endogenous growth literature [in particular, Romer (1986) Romer (1990) and Jones (1995) Jones (2003)], we consider a two-sector environment where two kinds of economic goods are generated: final goods, that can be either consumed or used as capital in the generation of new goods, and technology. Variable $k_{t}$ will define real per capita capital, which depreciates at a rate $\delta>0$, and $A_{t}$ will represent the technological level of the economy. The capital accumulation constraint is the following,

$$
\Delta k_{t}=A_{t} . f\left(k_{t}\right)-c_{t}-\delta . k_{t}, \Delta k_{t}=k_{t+1}-k_{t}, k_{0} \text { given. }
$$

In (2), the production function is assumed to exhibit constant marginal returns. Thus, this function may be interpreted as an endogenous growth production function, similar to the one in Rebelo (1991). We choose to work with the following explicit form for this function, $f\left(k_{t}\right)=\zeta . k_{t}, \zeta>0$. 
The second sector generates technology, a non rival good that can be simultaneously used in the production of physical goods and in the generation of additional technology. We consider decreasing but positive marginal returns in the accumulation of technological knowledge [as in Jones (2003) we may interpret this statement as translating the existence of positive intertemporal technology spillovers]. Furthermore, technology generation depends solely on the previously accumulated knowledge. Therefore, given a parameter $\phi \in(0,1)$, the technology production function is considered as a function $f^{A}\left(A_{t}\right)=A_{t}^{\phi}$.

In a homogeneous scenario regarding technological investment opportunities, the following dynamic rule reflects the accumulation of technological knowledge,

$$
\Delta A_{t}=g \cdot f^{A}\left(A_{t}\right)-\omega \cdot A_{t}, \Delta A_{t}=A_{t+1}-A_{t}, A_{0} \text { given. }
$$

In (3), parameter $g$ is a positive productivity parameter and $\omega$ is an obsolescence rate for technology.

Our attention will focus on a setup with research heterogeneity. This means the existence of various investment alternatives regarding technology production. We assume that the economy is populated by a large number of researchers and that there are alternative research activities $h=1, \ldots, H$. The distinction between research activities in our framework will be made considering different degrees of risk involved in the innovation process, i.e., all activities share the same expected outcome but diverse levels of volatility characterize the various possible outcomes. The heterogeneity will be translated through parameter $g$; we assume that different research projects imply distinct values for this technological productivity component. In this way, equation (3) splits in $H$ equations, each one representing the time evolution of the accumulation of technological knowledge regarding each specific innovation process,

$$
\Delta A_{h t}=g_{h t} \cdot f^{A}\left(A_{t}\right)-\omega \cdot A_{h t}, \Delta A_{h t}=A_{h t+1}-A_{h t}, A_{h 0} \text { given. }
$$

Note that in (4) the accumulation of knowledge through a project of type $h$ corresponds to the productivity of all the already existent knowledge when applied to type $h$ innovative activity; the obsolescence of this kind of technology contributes negatively to its accumulation.

For the productivity value $g_{h t}$ we now assume that $\left\{g_{h t}, t=1,2, \ldots\right\}$ is a Markov process. This Markov process is similar to the one in Kurz et al. (2003), i.e., the following dynamic rule is considered,

$$
\ln \left(g_{h t+1}\right)=\lambda \cdot \ln \left(g_{h t}\right)+\varepsilon_{h t+1}, \varepsilon_{h t} \sim N\left(0, \sigma_{h}^{2}\right) \text { iid }
$$

with $\lambda$ a positive parameter. Note that the only source of heterogeneity is the standard deviation of the normal distribution. Two possibilities regarding technological research will represent two different knowledge accumulation rates because the volatility associated with each project is not equal. Given different time paths for $g_{h t}$, we guarantee that the accumulation of $A_{h}$ through (4) differs among investment in technology decisions; furthermore, given that such accumulation process is dependent on a Markov process we will have stochastic time paths characterizing technology values and technology growth rates.

The index of technology available to the production of physical goods is an aggregate value, which may be thought as a weighted average of the technological level that results from each one of the $H$ research activities. Let $n_{h t}$ represent the share of researchers that at any time moment choose to follow the research strategy $h$; then we define

$$
A_{t}=\sum_{h=1}^{H} n_{h t} . A_{h t}
$$

The fractions $n_{h t}$ are updated in time according to a bounded rationality rule or a learning mechanism. Researchers compare their results with the results of alternative research strategies and change to the best strategy, but this does not happen instantly or permanently. The adaptive learning rule that 
is adopted follows the asset pricing literature that have introduced the concept of 'rational routes to randomness', namely Brock and Hommes $(1997,1998)$. This learning rule is based on discrete choice models, in the line of Manski and McFadden (1981) and Anderson et al. (1992), which implies the following value for the assumed share,

$$
n_{h t}=\frac{e^{\beta . a_{h t}}}{\sum_{i=1}^{H} e^{\beta . a_{i t}}}, \beta \geq 0, h=1, \ldots, H
$$

In expression (7), $\beta$ is an intensity of choice parameter. It represents the degree of rationality with which researchers choose to change the reallocation of their effort to another research project. If $\beta \rightarrow \infty$ the degree of rationality is maximum, that is, individuals change strategies immediately in the presence of better results than the ones obtained with the chosen strategy. For $\beta=0$, researchers will never change strategy independently of the obtained results. We assume that $\beta$ is a positive finite value, representing a bounded rationality behavior for researchers.

Variables $a_{h t}$ are performance measures or fitness functions that translate the past performance of the chosen research strategy. These functions have as a central property the fact that older observations are less relevant than recent observations [this follows a same kind of rule adopted in Barucci (1999) for the study of expectational stability in macroeconomic models with heterogeneous beliefs]. So, we consider a factor $\tau>0$ that discounts to the present past technological outcomes. Each $a_{h t}$ function is then the sum of all the past technology values until some present moment $T$, according to (8).

$$
a_{h t}=\sum_{t=0}^{T} A_{h t} \cdot \frac{1}{(1+\tau)^{T-t}}, h=1, \ldots, H
$$

We are now in conditions to define formally our model.

Definition 2.1 (Heterogeneous researchers two-sector model). The representative consumer of the economy controls the time path of consumption in order to maximize the sequence of utility values in (1). The maximization problem is subject to a capital accumulation constraint, (2), and to a series of $H$ technology generation rules, (4). Technological results vary according to a Markov process affecting technology productivity, (5), being innovation risk the source of heterogeneity. The number of researchers choosing an innovation strategy is determined by a bounded rationality rule, (7), where past results constitute the criteria underlying such choices, as indicated by (8). The level of technology that determines goods production is an average of the several technological achievements, as in (6).

\section{DYNAMICS AND STEADY-STATE PROPERTIES}

The analytical treatment of the optimal control problem in definition 2.1 does not allow to obtain completely unequivocal results. This is because the difference in volatility assumption implies that the same parameter values may give place to different time trajectories for the main variables of the model. Because all research projects have the same expected outcome, projects with high and low risk alternate as the ones that produce more technological knowledge in a totally random way, and so we will not be able to predict future results. In this section we study the dynamics of the model in the vicinity of the expected steady state. In the following section, we allow for volatility in research projects and making use of a numerical example we will display and discuss the unpredictability of technology and consumption long run growth rates. 
Definition 3.1 (Expected Steady State). Defining $E\left(g_{h t}\right)$ as the expected value of the stochastic variable $g_{h t}$, so that $E\left(g_{h t+1}\right)=E\left(g_{h t}\right)$, the expected steady state will be a long run locus in which the technology level is a constant value and the consumption-capital ratio is also constant. ${ }^{1}$

Having in mind definition 3.1, we can prove several propositions. To do this, we first encounter the optimality necessary conditions of the problem in definition 2.1. Consider a Hamiltonian function, a shadow-price for capital, $p_{k t} \geq 0$, and a set of co-state variables for each one of the technology variables, $p_{A h t} \geq 0, h=1, \ldots, H$. The current-value Hamiltonian function is

$$
\aleph\left(k_{t}, A_{h t}, c_{t}\right)=U\left(c_{t}\right)+p_{k t} \cdot\left[A_{t} \cdot f\left(k_{t}\right)-c_{t}-\delta \cdot k_{t}\right]+\sum_{h=1}^{H} p_{A h t} \cdot\left[g_{h t} \cdot f^{A}\left(A_{t}\right)-\omega \cdot A_{h t}\right]
$$

Optimality necessary conditions are:

$$
\begin{gathered}
\frac{\partial \aleph}{\partial c_{t}}=0 \Rightarrow c_{t}^{-\theta}=p_{k t} \\
\Delta p_{k t}=\rho \cdot p_{k t}-\frac{\partial \aleph}{\partial k_{t}} \Rightarrow \Delta p_{k t}=\left(\rho+\delta-\zeta \cdot A_{t}\right) \cdot p_{k t} \\
\Delta p_{A h t}=\rho \cdot p_{A h t}-\frac{\partial \aleph}{\partial A_{h t}} \Rightarrow \\
\Delta p_{A h t}=\left(\rho+\omega-\phi \cdot n_{h t} \cdot A_{t}^{-(1-\phi)} \cdot \sum_{i=1}^{H} g_{i t} \cdot p_{A i t}\right) \cdot p_{A h t}-p_{k t} \cdot n_{h t} \cdot f\left(k_{t}\right), h=1, \ldots, H \\
\lim _{t \rightarrow+\infty} p_{k t} \cdot \frac{1}{(1+\rho)^{t}} \cdot k_{t}=0 ; \lim _{t \rightarrow+\infty} p_{A h t} \cdot \frac{1}{(1+\rho)^{t}} \cdot A_{h t}=0
\end{gathered}
$$

[transversality conditions]

For the set of equations (10) to (13), we present proposition 3.2.

Proposition 3.2 (Sufficient Optimality Conditions). First order optimality conditions (10 to 13) are also sufficient, given the Arrow and Kurz (1970) theorem, if one assumes that the following condition holds,

$$
2 . \zeta . p_{k t} \cdot \sum_{h=1}^{H}\left(n_{h t} \cdot z_{0} \cdot z_{h}\right) \leq \phi .(1-\phi) \cdot A_{t}^{-(2-\phi)} \cdot \sum_{h=1}^{H}\left[p_{A h t} \cdot g_{h t} \cdot n_{h t} \cdot \sum_{j=1}^{H}\left(n_{j t} \cdot z_{h} . z_{j}\right)\right] \text {, }
$$

$\forall z_{0}, z_{1}, \ldots, z_{H} \in \mathbb{R}$

The following analysis assumes that the values of parameters and variables are such that condition (14) is satisfied. The proof of proposition 3.2 is presented in appendix A, in the end of the text.

Relation (10) can be used to change (11) into a dynamic equation relating to the growth path of consumption. We find a result that is common in endogenous growth literature, ${ }^{2}$

$$
\frac{\Delta c_{t}}{c_{t}}=\frac{1}{\theta} \cdot\left[\zeta \cdot A_{t}-(\rho+\delta)\right]
$$

\footnotetext{
${ }^{1}$ Note, furthermore, that under the assumption of equal productivity initial values, $g_{10}=g_{20}=\ldots=g_{H 0}$, the expected values will be identical in every time moment: $E\left(g_{1 t}\right)=E\left(g_{2 t}\right)=\ldots=E\left(g_{H t}\right)$. While this assumption may be relevant in an analysis of transitional dynamics outside the steady state vicinity, it is not important in the steady state neighbourhood analysis that follows, because for all $g_{h t}$ we verify that $\Delta E\left(g_{h t}\right)=E\left(g_{h t}\right)^{\lambda}-E\left(g_{h t}\right)$, and, thus, $\bar{E}(g)=1$ defines the long term equilibrium for any of the $H$ research projects.

${ }^{2}$ Note that this is an approximated result, which is reasonable only for small changes in $c_{t}$ over time (the result is exact in continuous time).
} 
The consumption growth rate in (15) would be a constant value if the technological level were constant. Since every research project is subject to decreasing marginal returns, the expected value of $A_{t}$ tends effectively to a long run steady state constant value, but the Markov process associated with the productivity of technological projects implies a consumption growth rate that would be around a constant value that does not, however, stabilize in such value. Periods of high and low volatility will alternate as the technological projects with high and low risk perform better, according to the learning process given by the bounded rationality mechanism. This fact will be highlighted in next section's example.

Relatively to the expected steady state and the dynamics in the expected steady state vicinity, these are characterized by the following propositions (the correspondent proofs are presented in appendix A).

Proposition 3.3 (Expected Steady State Existence and Uniqueness). The optimal control problem in definition 2.1 has a unique expected steady state as described in definition 3.1.

Let us define $\psi_{t} \equiv c_{t} / k_{t}$; the unique steady state mentioned in proposition 2 is the vector (see the proof in appendix),

$$
\left[\begin{array}{c}
\bar{\psi} \\
\bar{A}_{h}
\end{array}\right]=\left[\begin{array}{c}
(\zeta+1 / \theta) \cdot \bar{A}+\frac{\theta-1}{\theta} \cdot \delta+\frac{1}{\theta} \cdot \rho \\
{\left[\frac{\bar{E}(g)}{\omega}\right]^{1 /(1-\phi)}}
\end{array}\right]
$$

Expression (16) presents the steady state value of the consumption-capital ratio and the expected steady state value of a $h$ research project outcome. Note that every research project has a same steady state expected outcome since, as regarded earlier, the long run expected productivity of each project is the same $[\bar{E}(g)=1]$. Note also that $\bar{A}=\bar{A}_{h}, \forall h=1, \ldots, H$, given the definition of $A_{t}$ in (6).

Proposition 3.4 (Stability properties). The system relating to variables $\psi_{t}$ and $A_{h t}$ exhibits saddle-path stability in the expected steady state vicinity, for any $h=1, \ldots, H$.

Proposition 3.5 (Convergence properties). The system relating variables $\psi_{t}$ and $A_{h t}$ presents, in the expected steady state vicinity and for any $h=1, \ldots, H$, a saddle trajectory characterized by an increasing consumption-capital ratio in the presence of an increasing technological level.

The three previous propositions are common to the class of two-sector models with capital constant returns and technology decreasing returns. The difference is that we have considered initially that researchers are distributed by different projects. The notion of expected steady state eliminates the importance of the existence of diverse innovation strategies, because those were distinguished only through different risk parameters. So, the research projects will all perform the same (we expect this) and consequently there are no incentives to change behavior, that is, the expected values of the shares $n_{h t}$ will be constant values. The model was in this way reduced to a one-dimension technology model, in which $A_{t}=n_{1} \cdot A_{1 t}+\ldots+n_{H} \cdot A_{H t}$.

\section{GROWTH-PATHS: A NUMERICAL EXAMPLE}

The expected steady state notion reduces the heterogeneous agents model to a homogeneous setup. In this section we return to the model with different risk in technology investments and look to a numerical example, through which we perceive that the same parameter values imply an infinite set of possibilities for the technology and consumption long run growth rates.

It is important to clarify the following. The initial values of the productivity variables $g_{h t}, h=$ $1, \ldots, H$, are irrelevant in terms of long run results. When considering the expected steady state we have regarded that $\bar{E}\left(g_{h}\right)=1, \forall h$, independently of initial conditions. If one assumes that the Markov process plays a relevant role in the long run, as we do in this section, the average value of each productivity variable continues to be equal to one, while the volatility of the Markov processes varies for each $g_{h}$. As a consequence, the chaotic behavior we observe for the variables of the model is essentially 
the result of the bounded rationality mechanism that is adopted, but an important aspect must be taken into account - because the expected value of $E\left(g_{h}\right)$ is the same for all technological projects there is a co-existence of periods where a project performs better than others with periods where the reverse occurs. It is this process that allows the mechanism of discrete choice to produce the chaotic behavior that the endogenous variables of the model, namely consumption, display. If some $g_{h}$ process had a higher average than the others, the correspondent accumulated technology outcome would be systematically superior and this process would dominate all the others; hence, the chaotic behavior would give place to the Markov process of the dominant project. Therefore, we should stress that it is the average value of the Markov processes relating productivity that triggers the chaotic behavior we observe (if the average values are equal or not too different), and not the initial values of $g_{h t}$; long term results are not dependent on these.

The numerical example takes the following set of parameter values:

$$
\left[\phi ; \tau ; \omega ; \beta ; \sigma_{1} ; \sigma_{2} ; \zeta ; \lambda ; \rho ; \theta ; \delta\right]=[0.25 ; 0.05 ; 0.06 ; 1 ; 0.1 ; 0.01 ; 1 ; 0.9 ; 0.04 ; 20 ; 0.01]
$$

The initial values $g_{10}=g_{20}=1$ and $A_{10}=A_{20}=0.6$ are also considered. Note that in this example heterogeneity in research projects is limited to dimension 2: $H=2$. The only distinction between research projects is associated to the volatility parameter (ten times higher for research project 1 than for research project 2, meaning that the risk associated to activity 1 is considerably larger). To obtain reasonable results with this set of parameters (consumption growth rates around 3\%) we calibrate the model by considering that the productivity parameter in equations (4) is equal to $g_{h t} / 25$, with $g_{h t}$ the stochastic variables defined through the dynamic rule in (5).

The previous parameter values and initial states allow to present the long run time trajectories for the several variables in the model. We focus the attention on four time paths:

a) the stochastic productivity variables, $g_{1 t}$ and $g_{2 t}$;

b) the aggregate technology growth rate;

c) the share of researchers engaged in scientific/technological activity $1, n_{1 t}$;

d) the aggregate consumption growth rate.

The main feature of the results for the referred aggregates is that they change substantially each time the example is run. As stated in previous sections, the fact that any of the two projects can perform better in each moment of time implies that it is not known in anticipation which is the project that will attract more researchers; since the rule to change research strategies is an adaptive rule, the time trajectories can follow substantially different paths for the same parameters and initial values of variables.

In appendix B we present several time paths, for the previously mentioned variables. The first set of figures (figures 1 to 3 ) is a set of three possible realizations of the productivity variables $\left(g_{1 t}\right.$ and $\left.g_{2 t}\right)$ trajectories over time. As we expected, the two series alternate over time as the best result regarding research productivity. The main regularity is the one imposed by the heterogeneity source: the first series presents a well evident higher volatility. The series $g_{2}$ displays a lower research risk, but, as assumed, the two series present an equal expected outcome: $E\left(g_{1}\right)=E\left(g_{2}\right)=1$. The two time trajectories have differences for each one of the examples, given the stochastic component governing the Markov process. Nevertheless, there is a pattern: the higher volatility regarding the first research project, the same expected value, the reversion to the mean characteristic and the variability relating to the strategy that best performs are features present in any of the three first figures.

Figures 4 to 6 are the graphical representations of the growth rate of the technology variable. The technology variables, relating to each innovation project, evolve in time according to equation (4) and the aggregate technology variable is just an average of the technology results [remind (6)]. Thus, given 
the property of decreasing marginal returns, the long run value of this rate is, in the absence of random productivity, equal to zero. As displayed, the growth rate of $A_{t}$ fluctuates around a constant value. The important evidence is that there is not an identifiable pattern of evolution in time for this variable. The bounded rationality setup contributes to periods of high and low volatility to coexist in a perfectly unpredictable way. The only element in common among the lines in figures 4 to 6 is the zero expected value.

We now turn to the graphical representation of the share of researchers affected to each of the two R\&D projects. Figures 7 to 9 display the share of individuals working in knowledge creation that are associated with type 1 activities (symmetric lines would represent the share of individuals engaged in type 2 research activities). The adaptive learning process and the constant change in terms of the best performing strategy are the two key points explaining the absence of a pattern linking the three time paths in consideration. A same set of parameters gives place to a potentially infinite number of solutions for the time trajectory of $n_{t}$; furthermore, since there is not a productivity value that assumes itself as the best one for a long period of time, the variable under appreciation does not tend to stay near zero or near one for long periods of time, meaning this that one of the projects does not tend to concentrate all the researchers, and consequently researchers mobility is a frequent feature in our economic setup.

The growth rate of consumption is, in our model, the one in expression (15). We verify that this growth rate is a function of $A_{t}$ and of a set of parameters. In this way, the behavior over time of the growth rate of $c_{t}$ is qualitatively the same behavior of the technology aggregate variable. We have mentioned that $A_{t}$ has an expected constant long run value and thus the expected long run value of the consumption growth rate is also constant. From (16) is true that $\bar{A}=\left[\frac{E(g)}{\omega}\right]^{1 /(1-\phi)}=$ $\left(\frac{1}{25 \times 0.06}\right)^{1 /(1-0.25)}=0.582$ (note in this expression that the expected productivity value is divided by 25 , according to the calibration aspect referred in the beginning of the section). The expected long run value of consumption growth is $\bar{c}=\frac{1}{20} .[1 \times 0.582-(0.04-0.01)]=0.0266$. The consumption growth rate deviates from the average value in all the three presented figures [figures 10 to 12] but there is no regular pattern regarding the moments in which such deviations are more pronounced. The main feature is once more the absence of a predictable pattern. In this way, we have proposed an explanation to consumption growth unpredictability based on different degrees of uncertainty of the R\&D activities.

Two more items are subject to graphical representation in appendix B. These two items allow for a clearer picture about the unpredictability properties of our model. The first set of drawings [figures 13 to 15] relates to the graphical representation of $A_{2}$ (in the vertical axis) relatively to $A_{1}$ (in the horizontal axis). The second set [figures 16 to 18] is the set of stable trajectories between the consumption-capital ratio and each one of the two technology variables, according to the saddle-path expression derived in appendix A, (22).

Figures 13 to 15 represent the level of technology in research sector 2 for each level of technology in research sector 1 . The two lines that cross the graphic correspond to the expected steady state values $\bar{A}_{1}=\bar{A}_{2}=0.582$. The steady state point is the one in the intersection of the two lines. Volatility implies that there is not a unique equilibrium value but a large set of values that accumulate around the mentioned point. Confirming the information of the previous figures, larger deviations occur in the direction of higher technology values, and relatively high values for one technology variable tend to be accompanied by relatively high values of the other variable. The different shape of the line for each one of the three examples is evident in the figures.

Finally, figures 16 to 18 are saddle-path trajectories that obey equation (22) in appendix A. These trajectories indicate how the consumption-capital ratio, denoted by $\psi_{t}$, converges to the expected steady state point with the evolution of each one of the technology variables. The darker (and wider) lines are the ones relating to the pair of variables $\left(\psi_{t}, A_{1 t}\right)$ and the more compact (and clearer) lines respect to the relation between $\left(\psi_{t}, A_{2 t}\right)$ - the difference in volatility between the two technology productivity 
values is the reason for the difference in shape between the two time trajectories. These trajectories have positive slopes [according to (22)] but they are not straight lines; they are collections of points that gravitate around the steady state but where it is identifiable a tendency for a positive relation between variables: relatively high values of the technology variables imply, generally speaking, a tendency for $\psi_{t}$ higher values. Saddle-paths have different shapes but similar qualitative properties for simulations with a same set of parameters.

\section{DISCUSSION - HETEROGENEOUS RESEARCHERS AND ENDOGENOUS FLUCTUATIONS}

The present study intends to give an explanation about why macroeconomic time series display a not completely predictable pattern over time. Although this is a partial analysis, since it considers only one source of endogenous fluctuations (a bounded rationality mechanism linked to the R\&D investors decisions), it is sufficiently relevant to produce the kind of business cycles that we encounter in reality. Therefore, our most important argument goes beyond the simple heterogeneity of research projects; the argument is that differences in the way economic agents in some sector of activity conduct their businesses and take autonomous decisions may have widespread implications over the main economic aggregates time series, producing everlasting fluctuations where, in conventional models with fully rational agents, we would certainly find a steady long term behavior.

Traditionally in economic literature economic fluctuations can be interpreted relying on two strands of thought. One is the Keynesian view that output and other real aggregate variables move according to monetary and other aggregate demand disturbances, given the evidence of a sluggish adjustment in nominal prices and wages. The second interpretation is the one developed by Kydland and Prescott (1982), Long and Plosser (1983), Christiano and Eichenbaum (1992) and Baxter and King (1993), among many others, which has received the designation of real business cycle $(\mathrm{RBC})$ theory. Our explanation of fluctuations ignores any monetary phenomenon and thus it has an RBC flavour. Indeed, it is through the consideration of a random component linked to technology generation that fluctuations arise.

Nevertheless, there is a substantial difference between the presentation in the previous sections and RBC models. This distinction relates to: (1) there are no technological shocks; one just considers that different R\&D projects have different degrees of risk; (2) fluctuations are the direct result of absence of full rationality. Instead of considering that short run variations in aggregate output and consumption are the consequence of disturbances in technology (or other variables, like government purchases), we disaggregate the research activity in a large number of research projects and we take a simple but intuitive distinction between projects - the degree of uncertainty attached to R\&D projects varies, even for projects with a same expected return. The second feature, the bounded rationality setup, completes our fluctuation mechanism: if the researchers were capable of changing projects every time they found a better opportunity to make their activity more profitable, long term productivity time paths would be smooth and, given the link that our model establishes between this and other real variables like consumption, no cycles would be observable. But real life does not allow for such a perfect behavior. Researchers have different skills, and therefore the mobility of individuals between projects is reduced; many times contract obligations do not allow for a project to be abandoned at the date that it is desirable for the researcher to do so; the researcher does not have, in most circumstances, the sufficient knowledge / information about the outcome of other R\&D activities.

In the light of the arguments just presented we can frame our model in the following way: the heterogeneous researchers model is an RBC model in the sense that,

(a) the basic setup corresponds to an aggregate economic growth model (and growth and fluctuations are not different phenomena that can be studied independently from each other);

(b) fluctuations are (can be) the result of nondeterministic technology progress;

(c) no monetary phenomena are assumed; 
(d) without the stochastic component, the economy would not display long term fluctuations. and it adds two new features to such an interpretation of aggregate behavior; namely,

(e) heterogeneity of research activity;

(f) bounded rationality in research.

These two features allow for an RBC model where cycles are not the result of exogenous random shocks but the result of the intrinsic random nature of purposive R\&D, which is a more reasonable source of explanation for aggregate observed behavior in modern economies, where R\&D is the result of highly planned activities rather than something that happens from time to time as a consequence of what some brilliant mind is capable of conceiving.

Two important issues remain to be addressed in this discussion. The first relates to the nature of fluctuations: should these be considered as the result of deterministic or stochastic sources? The second is linked to the relation between the model's results and empirical evidence. Namely, we have used the technological heterogeneity to illustrate consumption long term patterns of evolution; are these the patterns that we observe in the real world?

In the heterogeneous researchers model, randomness is present. But it is not solely randomness that produces the kind of observed aggregate behavior. As Gomes (2005) explains, two elements are essential to produce unpredictable long run fluctuations in an adaptive learning context: (i) a bounded rationality mechanism; (ii) the individual time series must reflect a situation under which one of the outcomes is not systematically better or worse than the others. Therefore, randomness in productivity is important, but without bounded rationality and alternate best performances, the aggregate time series of consumption (and output) would just look like the path relating to the individual productivity variables. Despite the fact that our fluctuations are not just the result of randomness and that chaotic features are introduced through the rationality mechanism that is adopted, no strange dynamics would be produced, under our setup, without such random component. In this way, our model allows for an intermediate answer in what concerns the recent discussion about the nature of aggregate fluctuations. Contemporary literature on aggregate fluctuations emphasizes that these can be produced without the need for random variables. Assuming increasing returns and an open economy [Aloi et al. (2000)], externalities in production [Christiano and Harrison (1999)], or credit constraints [Caballé et al. (2004)], many authors are today able of replicating economic conditions under which aggregate variables behave in such a way that their time paths are impossible to predict, and this assuming deterministic scenarios. Cyclical and chaotic equilibria are systematically found in the cited studies for reasonable values of parameters.

As Bullard and Butler (1991) state: "Nonlinear dynamic models (...) offer the possibility of explaining economic phenomena in a purely endogenous manner, without resorting to ad hoc stochastic specifications." (page 8). The questions we may ask are whether, on one hand, should we look at aggregate data as produced only by deterministic factors? and, on the other hand, are stochastic specifications necessarily ad hoc? To the first question, the literature has developed a significant set of tentative answers. One of the most important is the strand of analysis that was initiated by Barnett and Chen (1988), who have proposed to test economic data in search for chaos. Finding chaos in economic time series would mean that fluctuations are indeed endogenous and that random variables are not important determinants of aggregate behavior. Although a consensus has not been produced so far, the large majority of studies in this field [e.g. Shintani and Linton (2003) and Serletis and Shintani (2003)] finds statistical results that cast relevant doubt over the usefulness of understanding economic data as chaotic.

If economists have serious doubts about the purely chaotic nature of economic series, it is reasonable to assume that fluctuations are produced by a mix of deterministic factors and a random component. This leads us to the above second question - where do we encounter natural sources of randomness, that are not arbitrarily imposed to generate strange and unpredictable dynamics? It seems 
that the model proposed in this paper gives a possible logical answer: the outcome of R\&D projects is unpredictable by nature, and it is the different degree of uncertainty of each project that triggers the economic behavior under which aggregate variables (in the case, consumption) display a chaotic kind of evolution over time. ${ }^{3}$

A last subject of discussion relates to the reasonability of the found consumption time series. Are these coherent with observed data and with economic interpretations of aggregate consumption behavior? Note the following features of our consumption results: (i) there is persistence over time, that is, the variability of consumption growth is low when compared with the variability of the aggregate technology variable; (ii) the consumption growth rate does not depart significantly from its expected value (changes in the consumption growth rate do not go beyond two percent points for the analyzed period, which includes the first thousand observations).

The relative constancy of consumption growth is characterized by Reis (2005), who, noting a well documented evidence, states that "one of the stylized facts about economic growth in the United States in the past century is that consumption, like income, has grown at an approximately constant rate." (page 5). European countries and developing economies also display relative consumption growth smoothness (when compared to other aggregate time series, like the ones relating technological progress; an observation of some OECD and IMF statistical data confirms this immediately).

In what concerns the coexistence of unpredictable periods of relatively high with relatively low consumption growth, note that this is a direct result of the way consumption growth is dependent on technological progress in our model. Nevertheless, there is theoretical reasoning that supports evidence at this level. For instance, Parker and Preston (2004) emphasize precautionary saving as a source of unpredictability in consumption - because people save to consume in the future, we may even observe countercyclical movements in expected consumption growth. In other analysis, Sommer (2003) explores the role of habit formation; habit formation means that consumers become addicted to the level of consumption they experienced in the past, and thus some persistence must be observed in consumption growth paths. In our results this persistence is present; high and low consumption growth rates are, alternatively, maintained over a few periods of time, independently of the technology index growth variability over time.

\section{CONCLUSIONS}

In an economy there are many types of R\&D activities. Some have a certain degree of certainty relating expected outcomes; others involve a considerable degree of risk: results may be the expected ones, much better than expected or, in opposition, much worse. Having this observation in mind, we have developed an optimal control problem for a representative consumer and a two-sector setup. The two assumed economic sectors were a final goods sector and a technological sector. The technological sector had the peculiarity of disaggregating research projects in a way that different uncertainty degrees in $R \& D$ projects were highlighted.

Combining the existence of distinct opportunities regarding innovation strategies with a rule of bounded rationality behavior for the agents engaged in the technology production process, we have attempted to put together an explanation for aggregate consumption growth paths volatility and unpredictability. It was shown that a same set of parameters and initial values of variables gives place to different consumption growth trajectories each time the example is concretized.

\footnotetext{
${ }^{3}$ By now the notion of chaos needs a clarification. If one wants to be rigorous, our long run result concerning the consumption time path is not chaos, in the sense that it is not the pure result of deterministic forces. A less restrictive notion of chaos allows for considering our time paths as such, namely we can adopt the notion of Lorenz (1997), page 119, to whom chaos is a situation where "a pair of initial values located arbitrarily close together may lead to completely different time series though they are generated by the same dynamical system".
} 
The developed model can be associated to the real business cycle literature, in the sense that it relies on technology stochasticity to explain real economic aggregates fluctuations (with a complete absence of nominal variables). The model's marginal contribution is that it allows for a result of compromise - endogenous fluctuations do not come exclusively from deterministic sources, but the source of randomness has economic meaning: R\&D projects have different degrees of associated uncertainty, and researchers tend to be dispersed along these projects.

\section{Bibliography}

Aloi, M., Dixon, H., \& Lloyd-Braga, T. (2000). Endogenous fluctuations in an open economy with increasing returns to scale. Journal of Economic Dynamics and Control, 24:97-125.

Anderson, S., De Palma, A., \& Thisse, J. (1992). Discrete choice theory of product differentiation. MIT Press.

Arrow, K. \& Kurz, M. (1970). Public investment, the rate of return, and optimal Fiscal policy.

Azariadis, C. \& Kaas, L. (2002). Asset Price Fluctuations without Aggregate Shocks. Technical report, University of California and University of Vienna.

Barnett, W. \& Chen, P. (1988). The Aggregation-Theoretic Monetary Aggregates are Chaotic and Have Strange Attractors: An Econometric Application of Mathematical Chaos. In W. A. Barnett, E. B. \& White, H., editors, Dynamic Econometric Modeling, pages 199-246. Cambridge University Press.

Barucci, E. (1999). Heterogeneous Beliefs and Learning in Forward Looking Economic Models. Journal of Evolutionary Economics, 9(4):453-464.

Baxter, M. \& King, R. (1993). Fiscal Policy in General Equilibrium. The American Economic Review, 83(3):315-334.

Becker, R. \& Tsyganov, E. (2002). Ramsey Equilibrium in a Two-Sector Model with Heterogeneous Households. Journal of Economic Theory, 105(1):188-225.

Brock, W. \& Hommes, C. (1997). A Rational Route to Randomness. Econometrica, 65(5):1059-1095.

Brock, W. \& Hommes, C. (1998). Heterogeneous Beliefs and Routes to Chaos in a Simple Asset Pricing Model. Journal of Economic Dynamics and Control, 22(8):1235-1274.

Brock, W., Hommes, C., \& Wagener, F. (2001). Evolutionary Dynamics in Markets with Many Trader Types. Technical report, CeNDEF, Amsterdam.

Bullard, J. \& Butler, A. (1991). Nonlinearity and Chaos in Economic Models: Implications for Policy Decisions. Technical Report 1991-002B, Federal Reserve Bank of St. Louis.

Caballé, J., Jarque, X., \& Michetti, E. (2004). Chaotic Dynamics in Credit Constrained Emerging Economies. Technical Report 121, Barcelona Economics.

Chiarella, C. \& He, X. (2002). An Adaptive Model on Asset Pricing and Wealth Dynamics with Heterogeneous Trading Strategies. Technical report, University of Technology.

Christiano, L. \& Eichenbaum, M. (1992). Current Real Business Cycle Theories and Aggregate Labor Market Fluctuations. American Economic Review, 82:430-450.

Christiano, L. \& Harrison, S. (1999). Chaos, Sunspots and Automatic Stabilizers. Journal of Monetary Economics, 44:3-31. 
De Grauwe, P. \& Grimaldi, M. (2002). The Exchange Rate and its Fundamentals: a Chaotic Perspective. Technical Report 639, CESifo.

Evans, G. W. \& Honkapohja, S. (2001). Learning and Expectations in Macroeconomics. Princeton University Press.

Gaunersdorfer, A., Hommes, C., \& Wagener, F. (2003). Bifurcation Routes to Volatility Clustering under Evolutionary Learning. Technical report, CeNDEF, Amsterdam.

Gomes, O. (2005). Volatility, Heterogeneous Agents and Chaos. The Electronic Journal of Evolutionary Modeling and Economic Dynamics, 3(1047):1-32.

Hommes, C. H., Sonnemans, J., Tuinstra, J., \& van de Velden, H. (2002). Expectations and Bubbles in Asset Pricing Experiments. Technical Report 02-05, CeNDEF, University of Amsterdam.

Jones, C. I. (1995). R \& D-Based Models of Economic Growth. Journal of Political Economy, 103(4):759-784.

Jones, C. I. (2003). Population and Ideas: A Theory of Endogenous Growth. In Aghion, P., Frydman, R., Stiglitz, J., \& Woodford, M., editors, Knowledge, Information, and Expectations in Modern Macroeconomics, pages 498-521, Princeton, New Jersey. Princeton University Press. Honor to Edmund S. Phelps.

Kurz, M. (1997a). Asset prices with rational beliefs. In Kurz, M., editor, Endogenous Economic Fluctuations: Studies in the Theory of Rational Belief, number 6 in Springer series in Economic Theory, chapter 9. Springer.

Kurz, M. (1997b). Asset Prices with Rational Beliefs. In Kurz, M., editor, Endogenous Economic Fluctuations: Studies in the Theory of Rational Belief, number 6 in Studies in Economic Theory, chapter 7, pages 211-250. Springer-Verlag, Berlin and New York.

Kurz, M. \& Beltratti, A. (1997). The Equity Premium is no Puzzle. In Kurz, M., editor, Endogenous Economic Fluctuations: Studies in the Theory of Rational Belief, number 6 in Studies in Economic Theory, chapter 11, pages 283-316. Springer.

Kurz, M., Jin, H., \& Motolese, M. (2003). Endogenous Fluctuations and the Role of Monetary Policy. In Aghion, P., Frydman, R., Stiglitz, J., \& Woodford, M., editors, Knowledge, Information, and Expectations in Modern Macroeconomics (in Honor of Edmund S. Phelps), pages 188-227. Princeton University Press, Princeton, New Jersey.

Kurz, M. \& Motolese, M. (2001). Endogenous uncertainty and market volatility. Economic Theory, 17(3):497-544.

Kurz, M. \& Schneider, M. (1996). Coordination and correlation in Markov rational belief equilibria. Economic Theory, 8(3):489-520.

Kydland, F. \& Prescott, E. C. (1982). Time to Build and Aggregate Fluctuations. Econometrica, 50(6):13451370.

Long, J. B. \& Plosser, C. I. (1983). Real Business Cycles. Journal of Political Economy, 91(1):39-69.

Lorenz, H.-W. (1997). Nonlinear dynamical economics and chaotic motion. Springer-Verlag, Berlin and New York, 2 edition.

Maliar, L. \& Maliar, S. (2001). Heterogeneity in Capital and Skills in a Neoclassical Stochastic Growth Model. Journal of Economic Dynamics and Control, 25(9):1367-1397. 
Manski, C. \& McFadden, D. (1981). Structural analysis of discrete data with econometric applications. MIT Press, Cambridge, Mass.

Negroni, G. (2003). Adaptive Expectations Coordination in an Economy with Heterogeneous Agents. Journal of Economic Dynamics and Control, 28:117-140.

Nourry, C. \& Venditti, A. (2001). Determinacy of equilibrium in an overlapping generations model with heterogeneous agents. Journal of Economic Theory, 96:230-255.

Parker, J. A. \& Preston, B. (2004). Precautionary Saving and Consumption Fluctuations. Technical report, Princeton University and Columbia University.

Rebelo, S. (1991). Long-Run Policy Analysis and Long-Run Growth. Journal of Political Economy, 99:500521.

Reis, R. (2005). The Time-Series Properties of Aggregate Consumption: Implications for the Costs of Fluctuation. Technical report, Princeton University.

Romer, P. (1990). Endogenous Technological Change. Journal of Political Economy, 98:S71-S102.

Romer, P. M. (1986). Increasing Returns and Long-Run Growth. The Journal of Political Economy, 94(5):1002-1037.

Sargent, T. J. (1993). Bounded rationality in macroeconomics. Clarendon Press, Oxford.

Serletis, A. \& Shintani, M. (2003). No evidence of chaos but some evidence of dependence in the U.S. stock market. Chaos, Solitons and Fractals, 17(2):449-454.

Shintani, M. \& Linton, O. (2003). Is There Chaos in The World Economy? A Nonparametric Test Using Consistent Standard Errors*. International Economic Review, 44(1):331-358.

Sommer, M. (2003). Habits, Sentiment and Predictable Income in the Dynamics of Aggregate Consumption. International Monetary Fund.

Tuinstra, J. \& Wagener, F. O. O. (2003). On Learning Equilibria. Technical report, CeNDEF, Amsterdam.

\section{A. PROOF OF PROPOSITIONS}

Proof of proposition 1. The Arrow and Kurz (1970) theorem may be applied to our optimal control problem as follows:

"Define $\aleph^{0}\left(k_{t}, A_{h t}\right), h=1, \ldots, H$ to be the maximum of $\aleph\left(k_{t}, A_{h t}, c_{t}\right)$, with respect to $c_{t}$. If $\aleph^{0}\left(k_{t}, A_{h t}\right)$ is concave in $k_{t}$ and $A_{h t}$, for given $p_{k t}$ and $p_{A h t}$, then the necessary conditions (10) to (13) are also sufficient."

Condition (10) allows to write function (17).

$$
\begin{aligned}
& \aleph^{0}\left(k_{t}, A_{h t}\right)= \\
& U\left(p_{k t}^{-1 / \theta}\right)+p_{k t} \cdot\left[A_{t} . f\left(k_{t}\right)-p_{k t}^{-1 / \theta}-\delta . k_{t}\right]+\sum_{h=1}^{H} p_{A h t} .\left[g_{h t} . f^{A}\left(A_{t}\right)-\omega \cdot A_{h t}\right]
\end{aligned}
$$

We want to averiguate if $\aleph^{0}$ is a concave function. It is concave if the correspondent Hessian matrix is negative semidefinite, for all positive $k_{t}$ and $A_{h t}$. The Hessian matrix is a $(H+1) \times(H+1)$ squared 
matrix of the form,

$$
\mathbf{H}\left(\aleph^{0}\right)=\left[\begin{array}{c:ccc}
\frac{\partial^{2} \aleph^{0}}{\partial k_{t}^{2}} & \frac{\partial^{2} \aleph^{0}}{\partial k_{t} \partial A_{1 t}} & \cdots & \frac{\partial^{2} \aleph^{0}}{\partial k_{t} \partial A_{H t}} \\
\hdashline \frac{\partial^{2} \aleph^{\sigma}}{\partial A_{1 t} \cdot \partial k_{t}} & \frac{\partial^{2} \aleph^{\sigma}}{\partial A_{1 t}^{2}} & \cdots & \frac{\partial^{2}{ }^{2}-}{\partial A_{1 t} \partial A_{H t}} \\
\vdots & \vdots & \ddots & \vdots \\
\frac{\partial^{2} \aleph^{0}}{\partial A_{H t} \cdot \partial k_{t}} & \frac{\partial^{2} \aleph^{0}}{\partial A_{H t} \partial A_{1 t}} & \cdots & \frac{\partial^{2} \aleph^{0}}{\partial A_{H t}^{2}}
\end{array}\right]
$$

Proceeding with the computation of the matrix elements, one gets,

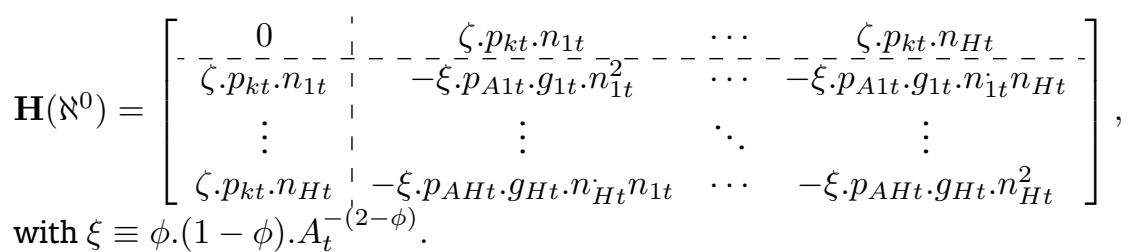

To find out if (18) is a negative semidefinite matrix, we take the correspondent definition: a $(H+1) \times$ $(H+1)$ matrix is classified as negative semidefinite if for all vectors $\mathbf{z}=\left[\begin{array}{lll}z_{0} & z_{1} \ldots z_{H}\end{array}\right] \in \mathbb{R}^{H+1}$, the condition $\mathbf{z}^{T} . \mathbf{H}\left(\aleph^{0}\right) \cdot \mathbf{z} \leq 0$ is satisfied. The condition is verified for combinations of parameters and variables that make (14) true. Therefore, necessary conditions are not universally sufficient; they are sufficient under condition (14).

Proof of proposition 2. Consider the set of equations (4). Because there is decreasing marginal returns in the production of technology, the technology variables will tend to long run constant values, and thus $\Delta A_{h t}=0$ defines a steady state with a constant $\bar{A}$ value. Furthermore, because, under definition (2), $E\left(g_{t+1}\right)=E\left(g_{t}\right)^{\lambda}$, one observes that $\bar{E}(g)=1$ defines the same steady state point, for every R\&D project. As a consequence, to determine the long run expected value of $A_{t}$, we substitute in (4) $g_{h t}$ by 1. Therefore, $\Delta A_{h t}=0 \Rightarrow \bar{A}_{h}=\omega^{-1 /(1-\phi)}, \forall h$, and $\bar{A}=\bar{A}_{h}$.

As regarded, there is a unique steady state level for technology. In what concerns consumption and capital variables, expression (15) indicates that consumption grows at a constant rate in the steady state, given the constant value of $\bar{A}$. Relatively to capital, equation (2) implies that consumption and capital must grow at a same steady state rate, and thus a variable $\psi_{t}=c_{t} / k_{t}$ will grow at a constant long run growth rate. The equation that reflects the time evolution of this variable is

$$
\Delta \psi_{t}=\left[\psi_{t}-\left(\zeta+\frac{1}{\theta}\right) \cdot A_{t}-\frac{\theta-1}{\theta} \cdot \delta-\frac{1}{\theta} \cdot \rho\right] \cdot \psi_{t}
$$

The unique steady state value of the consumption-capital ratio is $\Delta \psi_{t}=0 \Rightarrow \bar{\psi}=\frac{\zeta+1 / \theta}{\omega^{1 /(1-\phi)}}+\frac{\theta-1}{\theta} . \delta+$ $\frac{1}{\theta} \cdot \rho$.

The pair $(\bar{A}, \bar{\psi})$ is the unique steady state point of the system.

Proof of proposition 3. Saddle-path stability implies a Jacobian matrix with some (but not all) eigenvalues in the interval $(-2,0)$, since the system is defined in terms of first differences. Considering the several $A_{h t}$ variables, the following is a linearized version of the system composed by equations (4) 
and (19), in the vicinity of the steady state. Note that the matrix in the system is the Jacobian matrix.

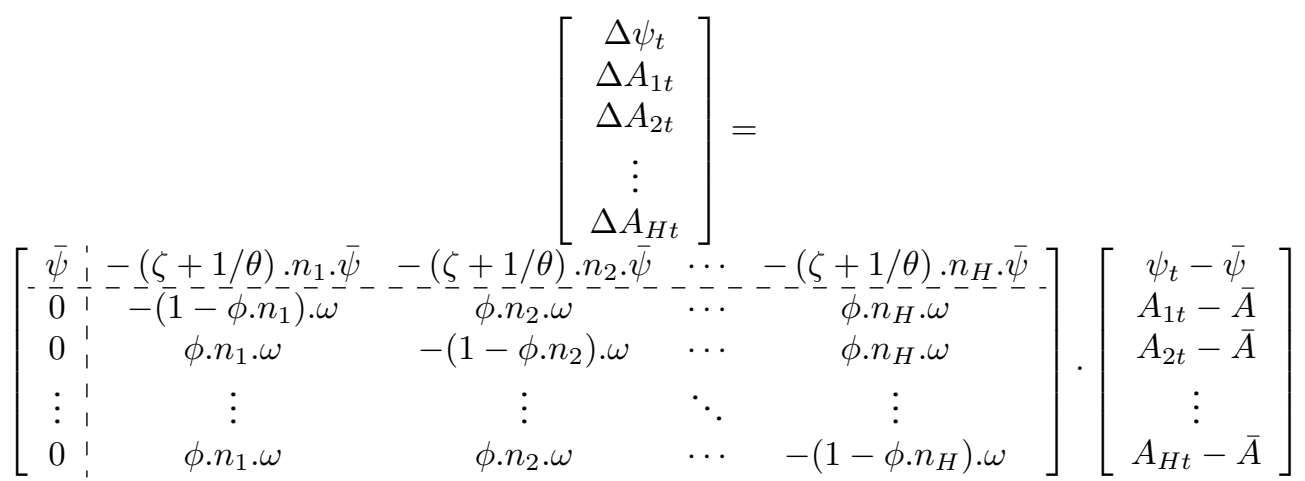

The Jacobian matrix in $(20)$ is a $(H+1) \times(H+1)$ squared matrix with trace $=\bar{\psi}-\omega \cdot(H-\phi)$ and determinant $=\bar{\psi} \cdot(-\omega)^{-1} \cdot(1-\phi)$. Computing eigenvalues, these are $\eta_{1}=\bar{\psi}>0, \eta_{2}=-\omega \cdot(1-\phi)<$ 0 and $\eta_{3}=\ldots=\eta_{H+1}=-\omega<0$. As a result, there is one positive eigenvalue corresponding to the one-dimensional unstable arm of the system and $H$ negative eigenvalues (that are smaller than 1 in absolute value), and thus the stable trajectory has dimension $H$. Because there are simultaneously stable and unstable trajectories, the equilibrium is defined by saddle-path stability.

Proof of proposition 4. Each one of the negative eigenvalues $\eta_{2}$ to $\eta_{H+1}$ has an associated eigenvector. These $H$ eigenvalues compose a matrix from which we can derive the slope of the stable trajectory. For

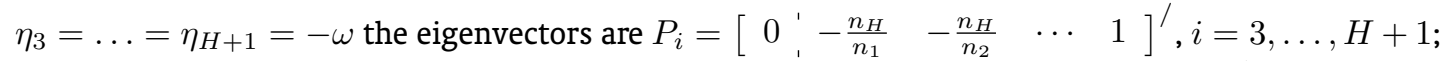
for $\eta_{2}$ we have the following eigenvector: $P_{2}=\left[\begin{array}{llllll}\frac{(\theta-1) \cdot \bar{\psi}}{\theta \cdot[\bar{\psi}+\omega \cdot(1-\phi)]} & 1 & 1 & \cdots & 1\end{array}\right]^{\prime}$. The matrix $P=\left[\begin{array}{llll}P_{2} & P_{3} & \cdots & P_{H+1}\end{array}\right]$ is a $(H+1) \times H$ matrix that can be divided in two; the slope of the stable trajectory is given by $-\pi . \Pi^{-1}$, where $\pi$ is the first line of $P$ and $\Pi$ is the square matrix composed by the lines 2 to $H+1$ of $P$. Some computation leads to a vector $\left[\begin{array}{llll}n_{1} & n_{2} & \cdots & n_{H}\end{array}\right]$ as the first line of $\Pi^{-1}$. This is the only line of the matrix that one needs to calculate the slope results, because only the first element of $\pi$ is different from zero. We have then,

$$
\text { Slope of the stable trajectory }=\frac{\theta-1}{\theta} \cdot \frac{\bar{\psi}}{[\bar{\psi}+\omega \cdot(1-\phi)]} \cdot\left[\begin{array}{llll}
n_{1} & n_{2} & \cdots & n_{H}
\end{array}\right]
$$

The slope of the stable trajectory is a set of positive values, given the constraints over parameter values that were established. Therefore, the stable trajectory is defined as

$$
\psi_{t}-\bar{\psi}=\frac{\theta-1}{\theta} \cdot \frac{\bar{\psi}}{[\bar{\psi}+\omega \cdot(1-\phi)]} \cdot\left[n_{1} \cdot\left(A_{1 t}-\bar{A}\right)+n_{2} \cdot\left(A_{2 t}-\bar{A}\right)+\ldots+n_{H} \cdot\left(A_{H t}-\bar{A}\right)\right]
$$

From (22) it is understandable that a convergence to the steady state through increasing values of technology levels implies that the consumption-capital ratio will also exhibit an increasing behavior. This result is common to this kind of models and it is obvious and intuitive: a higher technology level means that a final good can be produced with lower quantities of capital and thus a higher share of produced final goods can be directed to consumption. Note that (22) is equivalent to (23), given the definition of aggregate technology level,

$$
\psi_{t}=\bar{\psi}-\frac{\theta-1}{\theta} \cdot \frac{\bar{\psi}}{[\bar{\psi}+\omega \cdot(1-\phi)]} \cdot \bar{A}+\frac{\theta-1}{\theta} \cdot \frac{\bar{\psi}}{[\bar{\psi}+\omega \cdot(1-\phi)]} \cdot A_{t}
$$

Result (23) would be the one obtained directly if we had constructed the linearized model for the system $\left(\psi_{t}, A_{t}\right)$. 


\section{B. NUMERICAL EXAMPLE TIME TRAJECTORIES}

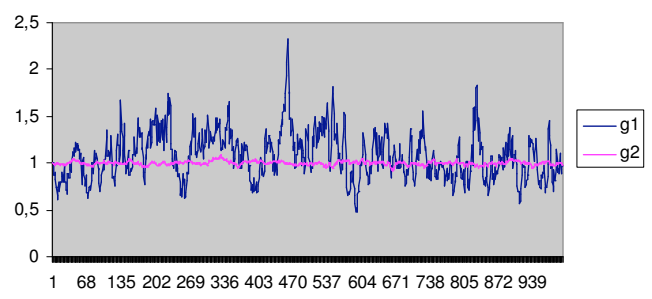

Figure 1 - Productivity variables time paths (example 1)

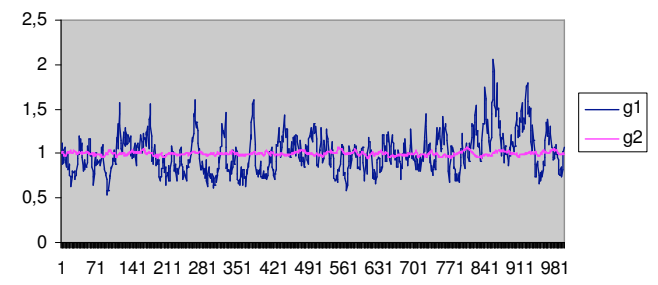

Figure 3 - Productivity variables time paths (example 3)

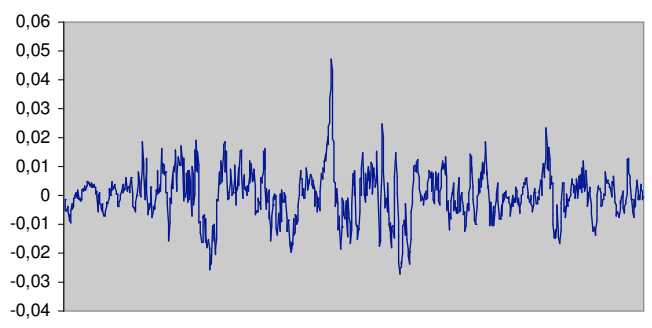

Figure 4 - A growth rate (example 1)

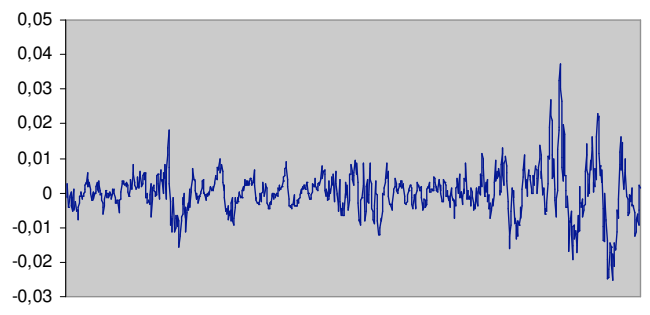

Figure 6 - A growth rate (example 3)

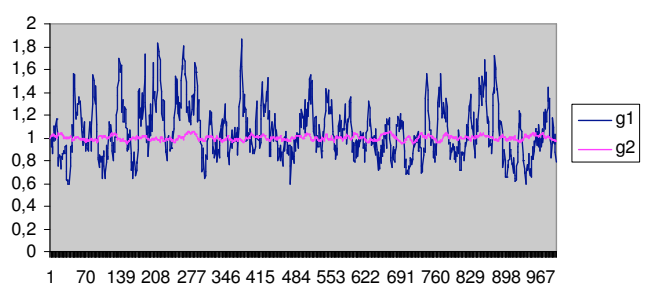

Figure 2 - Productivity variables time paths (example 2)

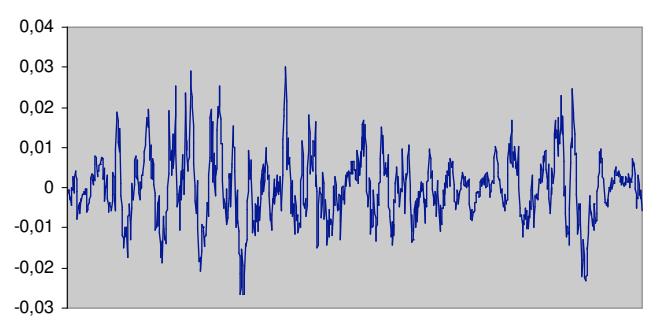

Figure 5 - A growth rate (example 2) 


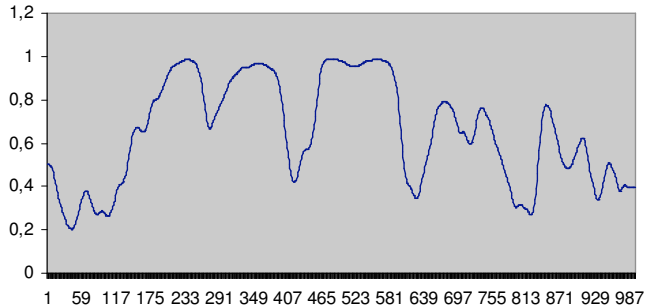

Figure 7 - n1 (example 1)

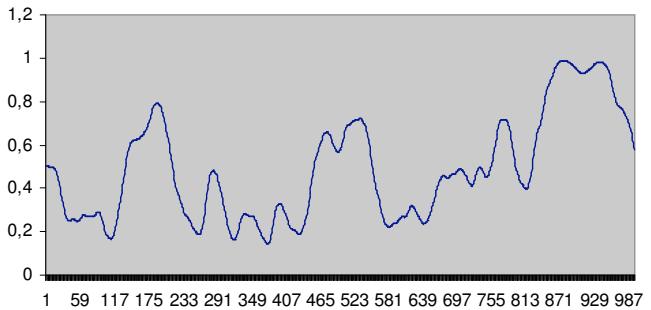

Figure $9-\mathrm{n} 1$ (example 3)

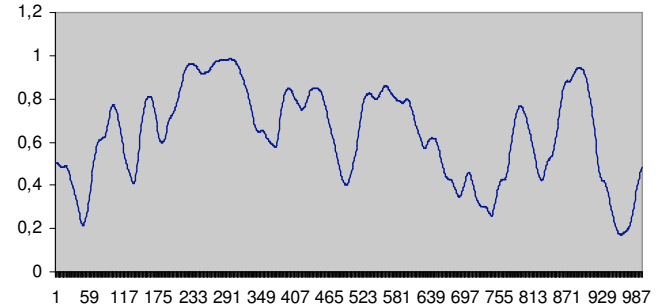

Figure 8 - n1 (example 2)

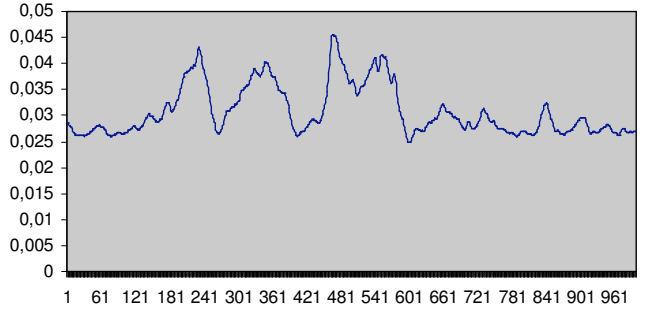

Figure $10-c$ growth rate (example 1 )

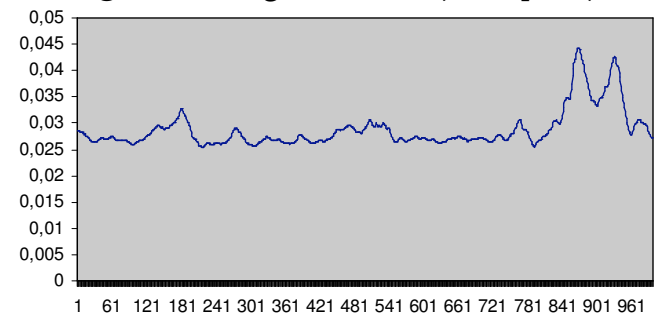

Figure $12-c$ growth rate (example 3)

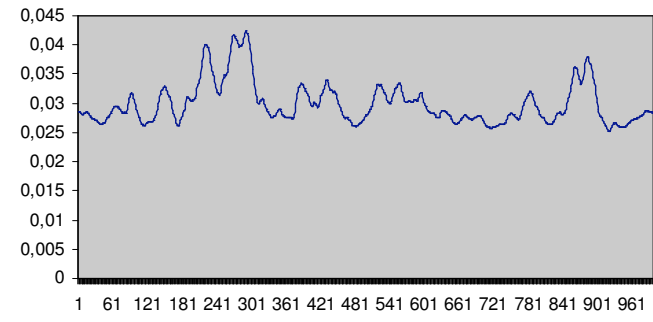

Figure 11 - c growth rate (example 2) 


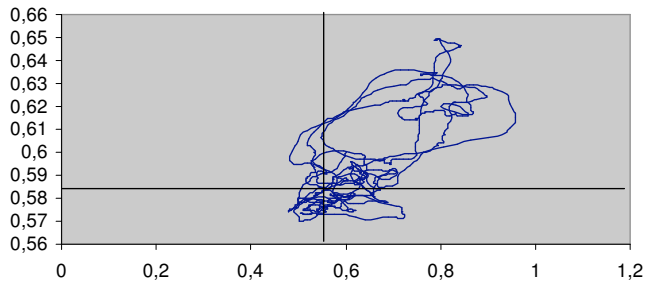

Figure 13 - Relation between technology levels (example 1)

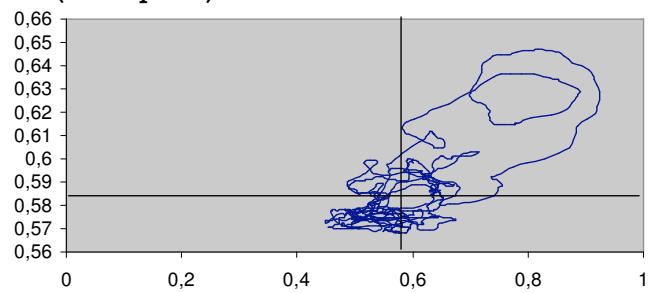

Figure 15 - Relation between technology levels (example 3)

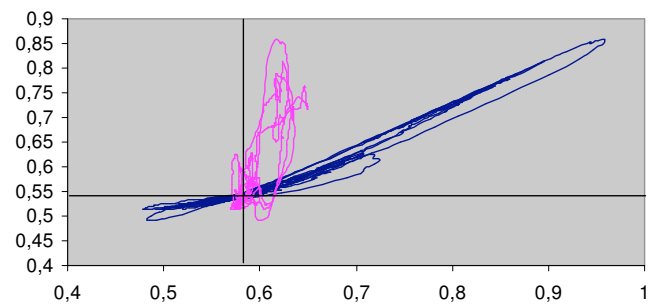

Figure 16 - Stable trajectories (consumptioncapital ratio / $A 1, A 2$ ) (example 1)

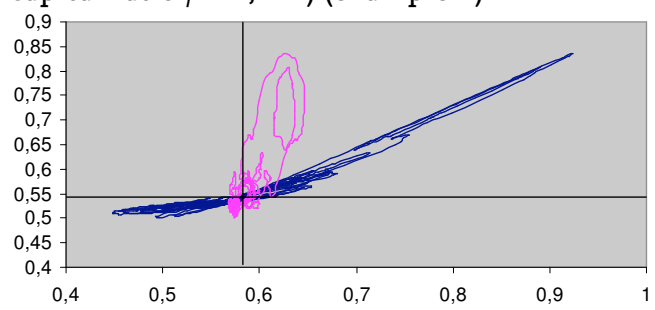

Figure 18 - Stable trajectories (consumptioncapital ratio / $A 1, A 2$ ) (example 3)

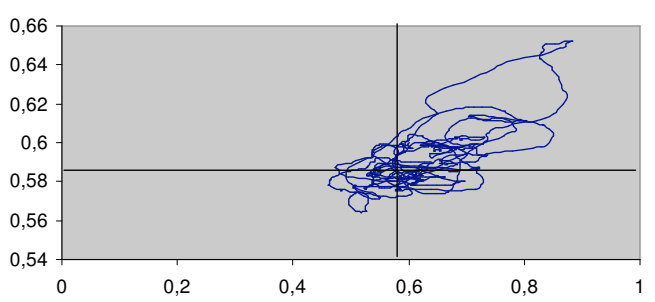

Figure 14 - Relation between technology levels (example 2)

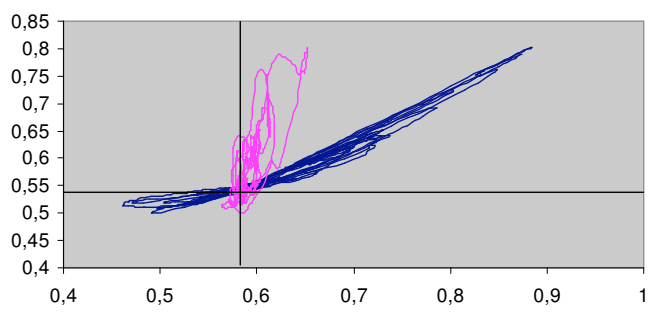

Figure 17 - Stable trajectories (consumptioncapital ratio / $A 1, A 2$ ) (example 2) 